\title{
Mood as an Affective Component for Robotic Behavior with Continuous Adaptation via Learning Momentum
}

\author{
Sunghyun Park, Lilia Moshkina, Ronald C. Arkin
}

\begin{abstract}
The design and implementation of $\operatorname{mood}$ as an affective component for robotic behavior is described in the context of the TAME framework - a comprehensive, time-varying affective model for robotic behavior that encompasses personality traits, attitudes, moods, and emotions. Furthermore, a method for continuously adapting TAME's Mood component (and thereby the overall affective system) to individual preference is explored by applying Learning Momentum, which is a parametric adjustment learning algorithm that has been successfully applied in the past to improve navigation performance in real-time, reactive robotic systems.
\end{abstract}

\section{INTRODUCTION}

$\mathrm{W}$ E humans have a complex affective system in which various elements come together in an intricate interplay to govern how we feel and behave. Personality predisposes us to have consistent behaviors in general over an extended period of time [1]. We are influenced by mood that can be cyclic or situational in nature [2]. We respond instantaneously to certain situations through our emotional reactions [3], and we also maintain an attitude towards certain objects [4].

Given our strong propensity in anthropomorphizing even inanimate objects around us [5], robots possessing a mature affective system can only help in capitalizing on this tendency for a more natural and effective interaction.

The focus of this paper is on generation and adaptation of Mood, one of four affective components within an integrative framework of time-varying affective robotic behavior, TAME, described earlier in Moshkina et al. [6]-[8]. The Mood component is designed with human-robot interaction in mind and provides a method for continuously adapting robotic moods (and thereby the overall affective system) to individual human preferences through application of Learning Momentum [9]-[12]. The latter is a parametric adjustment learning algorithm that has been successfully applied in the past to improve navigation performance in real-time, reactive robotic systems.

Manuscript received June 11, 2010. This work was supported by Samsung Electronics Co., Ltd. under Master Research Agreement number 669 with Georgia Tech Research Corporation (GTRC).

S. Park is with the Mobile Robot Laboratory, College of Computing, Georgia Institute of Technology, Atlanta, GA 30332, USA (phone: 404-993-5111; e-mail: gte246z@mail.gatech.edu).

L. Moshkina and R. Arkin are with the Mobile Robot Laboratory, College of Computing, Georgia Institute of Technology, Atlanta, GA 30332, USA (e-mail: \{lilia, arkin\}@cc.gatech.edu)

\section{RELATED WORK}

Although research in modeling affective systems abounds in the social robotics field [13], mood has received relatively little attention due to its subtle influence when compared to the personality traits and emotions. Not distinguishing mood as a separate affective element often occurs in this literature, and the term is sometimes even interchangeably and mistakenly used to mean emotions.

Among the work that actually differentiates mood as a separate affective element, there is Breazeal's robot Kismet [14], which uses its drive system to achieve a long-term affective state separate from its emotion system. Although Kismet's drive system may be used to simulate mood to some extent, it cannot be considered an explicit or complete mood element. In Itoh et al.'s mental model [15] for their humanoid robot WE-4RII, mood consists of the Pleasantness and Activation dimensions. Itoh et al.'s mood model is limited in that the Pleasantness dimension is influenced only by emotions, and the Activation dimension captures only a single biological rhythm as a whole. There is also Kubota's emotional model [16] for a pet robot in which the concepts of emotion, mood and feeling are treated separately. Here, however, only emotions have influence over mood as well, and cyclic characteristics of mood are not noted. Gockley's affective model for social robots [17] has a separate mood component that is associated with the emotion and attitude components. Mood in Gockley's model is determined by "life" events, and the storyline is arbitrarily encoded by the dramatic writers.

Regarding parametric adaptation, Learning Momentum has been applied in the past for robots reactively navigating through obstacles and in various environments. It has been shown to effectively adapt the behavioral control parameters in real-time for improved performance, both in the context of single [9], [10] and multi-robot [12] scenarios.

\section{MOOD COMPONENT}

The Mood component is one of four affective phenomena modeled in the TAME framework, with the others being personality Traits, affective Attitudes, and Emotions. Compared to our earlier work [6]-[8], this component has been redesigned to specifically target human-robot interaction by providing a mechanism for the robot to learn mood patterns that are more congruent with human partners. Also, with only minor changes (explained below), it has the potentials of being a flexible, comprehensive and independent 
Mood component (outside of the TAME framework) that can be easily integrated into other systems in need of this affective element.

\section{A. Psychological Foundations}

In the robotics community, mood is often confused with emotions, but there are strong psychological grounds giving mood the status of a separate affective construct independent of emotions. In particular, mood and emotions are known to be different in their duration and object specificity. While mood's influence falls in a longer span of time providing an affective background, emotions are understood to be "phasic perturbations on this background activity" [18]. Watson et al. [2] hold a similar view and depict mood as a "stream of affect," where a continuous affective state varies with time. Concerning object specificity, mood is seen to have a global and diffuse characteristic whereas emotions are narrow and specific in that respect [19]-[20]. In our everyday life, circumstances that cause emotional elicitations are relatively rare [2], and without a separate affective element of mood, it is not possible to capture the vicissitudes of affective experience that we undergo for most of the time; hence the importance of the inclusion of this affective element.

Mood is often represented along the two dimensions of Positive Affect and Negative Affect. Positive Affect is related to the level of pleasure and enthusiasm while Negative Affect is related to how much an individual is upset or distressed [21]. Isen [22] suggests that the two dimensions are independent of each other, meaning that a certain level of Positive Affect is not necessarily the inverse of the same level of Negative Affect.

From vast relevant literature, Watson et al. [2] summarize multiple factors that are believed to influence our overall mood. Some factors are cyclic in nature, and different cyclic patterns can result from different lifestyle, sociocultural, and biological rhythms. Such patterns can be diurnal, weekly, seasonal, etc. There are also situational and environmental factors influencing mood, such as specific causal events or external stimuli.

Individual differences in mood can be explained in part by different personality traits. In particular, dimensions of the Five-Factor Model of personality [1], [23], namely, Openness, Conscientiousness, Extroversion, Agreeableness, and Neuroticism, have been found to correlate with Positive and Negative Affect [2].

Lastly, on the relationship between emotions and mood, Ekman [24] suggests that mood can be generated when a person goes through an intense experience of a particular emotion for many times within a short period of time.

\section{B. Representation and Generation of Moods}

A separate mood type (abbreviated as a mood or moods for multiple mood types) is defined for each possible factor influencing mood generation. That is, there can be one mood capturing the diurnal biological rhythm and another mood that depends on the weather.

Given the correlation between the dimensions of mood and

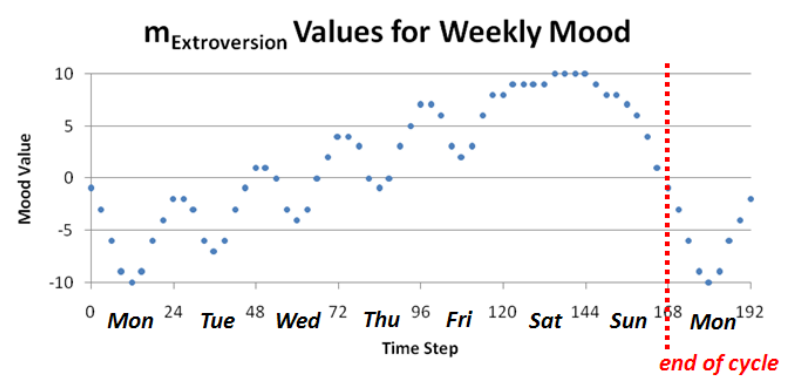

Fig. 1. An example cyclic workweek mood. Mood intensity values are displayed for the Extroversion dimension of personality with strong positive affect on the weekend.

the dimensions of personality, each mood is represented separately along each dimension of the Five-Factor Model as follows:

$$
\overrightarrow{\operatorname{mood}}=\left[m_{i}\right]
$$

where $i$ is each dimension of the Five Factor Model, $m_{i}$ is the mood intensity value for each dimension $i$, and $-10 \leq m_{i} \leq 10$ (-10 signifies extreme Negative Affect, 0 signifies absence of mood, and 10 signifies extreme Positive Affect).

For simplicity, Positive and Negative Affects are represented along a single dimension ranging from negative to positive real numbers and produce a positive or negative bias to each personality dimension. This bias, in turn, evokes adjustments in the behavioral parameters and emotion generations in the TAME framework [6]-[8].

An alternative representation of mood may be drawn along the dimension of Positive Affect and Negative Affect. This would decouple the relationship between personality and mood, rendering the Mood component more independent and easing its individual use and integration into other systems and architectures in need of this specific affective element.

There are two broad categories of mood: cyclic or circadian (e.g. based on time of the day, day of the week, seasons, etc.) and environmentally or situationally induced.

\section{Cyclic (Circadian) Moods}

The cyclic moods, in particular, have been introduced to allow the generation of mood-congruent robotic behaviors for multiple users. The patterns in cyclic moods are flexibly formulated using a configuration file, which is read at system run-time. For each cyclic mood, the file contains information such as its type, time step (hours, days of the week, days of the month, months, or years), the length of cycle, etc. The mood intensity value for each personality dimension is defined for an arbitrary number of time step points. If a time step point does not have any defined values, they are computed either using interpolation or a step function, depending on which option is selected in the file. When moving from one time step point to the next, the transition is made smooth through a weighted average filter.

Moods that have relatively definite and undeviating patterns, such as biological (endogenous) circadian rhythms, 


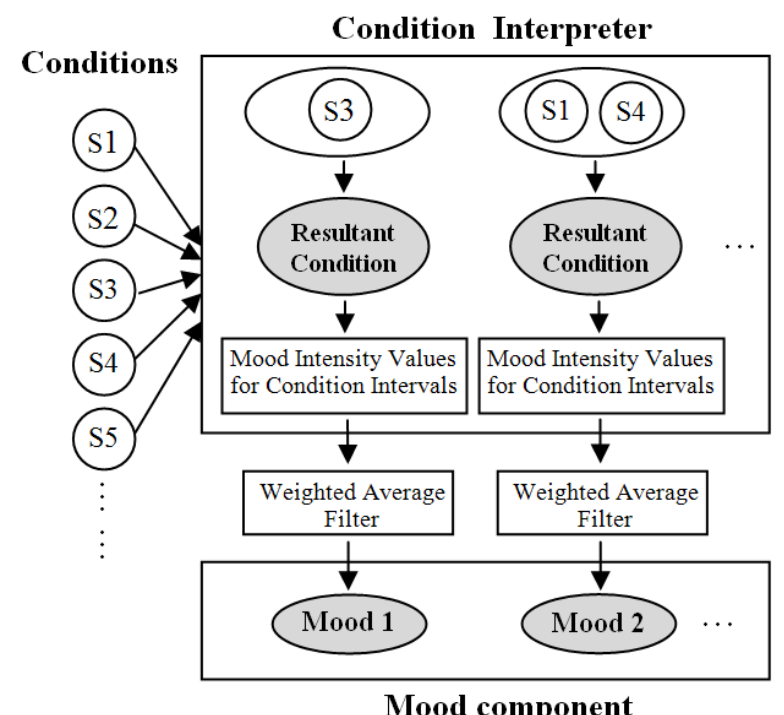

Mood component

Fig. 2. The process of how situational and environmental moods obtain their mood intensity values.

are appropriate candidates to be formulated with this scheme. The extent of flexibility is substantial since cyclic moods differ from individual to individual. An example workweek mood (for Extroversion) with hourly time steps is shown in Fig. 1. This method allows cyclic adjustment of the mood to match (entrain to) a particular user.

\section{Situational and Environmental Moods}

Situational and environmental moods depend on what is perceived in the environment (sensory) as well as how something is perceived (cognitive). Each piece of such information is termed a condition for moods, and the Mood component receives the strength value for each condition. For example, among various condition information that is streaming into the Mood component, assume that the "weather" mood is affected by the specific conditions of heat, coldness, sunniness, cloudiness, thunders, etc. The robot's sensors would gauge each condition level on the scale of 0 to 10 and relay the information to the Mood component.

Each situational and environmental mood is defined using a configuration file, which also allows specifying whether or not each incoming condition has any influence on the mood. All of the relevant and present conditions for a certain mood are taken together to yield an averaged influence:

$$
R C_{i}=S_{i} \cdot \frac{\sum_{j=1}^{N}\left(c_{j} \cdot s_{j}\right)}{N}
$$

where $R C_{i}$ is the resultant condition strength value for mood $i$, $S_{i}$ is the overall scaling factor for $R C_{i}, N$ is the number of relevant conditions for mood $i$ that are currently present, $c_{j}$ is the strength value for condition $j$, and $s_{j}$ is the scaling factor for $c_{j}$.

Using the configuration file, an arbitrary number of intervals of the resultant condition strength value can be defined for the mood intensity values along the five different dimensions. Depending on the interval into which the resultant condition strength value falls, the mood would obtain the corresponding intensity values as defined in the file. Changes in the mood intensity values due to interval transitions are smoothed with a weighted average filter. An illustrative description of this process is shown in Fig. 2.

\section{E. Resultant Mood and Its Influence}

All of the moods are combined together to make the resultant mood in the following manner:

$$
\operatorname{mood}_{\text {resultant }, j}=\frac{\sum_{i=1}^{N}\left(w_{i} \cdot m_{i, j} \cdot d p_{i, j}\right)}{\sum_{i=1}^{N} w_{i}}
$$

where mood $_{\text {resultant }, j}$ is the resultant mood intensity value for personality dimension $j, N$ is the total number of moods, $w_{i}$ is the strength (weight) of mood $i$ in computing the resultant mood, $m_{i, j}$ is the intensity value from mood $i$ for personality $j$, and $d p_{i, j}$ is the dependency information of mood $i$ for personality $j$.

The strength of each mood $\left(w_{i}\right)$ and its dependency $\left(d p_{i, j}\right)$ information are defined using the configuration file for maximum flexibility: $w_{i}$ can range from 0 to $\infty$, and $d p_{i, j}$ has a value in $\{-1,0,1\}$, where -1 signifies inverse influence, 0 signifies absence of influence, and 1 signifies direct influence.

Once the resultant mood is computed, it introduces bias to personality:

$$
P M_{i}=P_{i}+\mu \cdot \operatorname{mood}_{\text {resultant }, i}
$$

where $P M_{i}$ is the new, biased value for personality dimension $i, P_{i}$ is the original base value for personality $i, \mu$ is a scaling factor, and mood $_{\text {resultant }, i}$ is the resultant mood intensity value for personality $i$.

A bounding mechanism is in place to make sure the influence of moods is kept within certain limiting thresholds as defined in the configuration file.

As described, the Mood component introduces bias to personality, which is a component in TAME that determines important parameters in generating emotions, namely, Fear (F), Disgust (D), Anger (A), Sadness (S), Joy (J), and Interest (I). To account for the psychological studies showing emotions' influence on mood, the following equation can be used:

$$
m_{i}= \begin{cases}m_{i, \text { base }}+s \cdot E_{k}, & \text { if } E_{k} \in\{I, J\} \\ m_{i, \text { base }}-s \cdot E_{k}, & \text { if } E_{k} \in\{F, D, A, S\}\end{cases}
$$

where $i$ is each dimension of personality, $m_{i, b a s e}$ is the base mood intensity value for $i, s$ is a scaling factor, and $E_{k}$ is the currently present (or dominant) emotion.

For further details on the resulting direct and indirect influences within the TAME framework and on the associated behavioral parameters, the reader is referred to [6]-[8]. 


\section{LEARNING MOMENTUM}

Learning Momentum [9]-[12] can be thought of as a simple form of reinforcement learning for online and continuous parametric adjustments. The basic concept behind it is that you should keep on doing what you are doing if the performance is good, and maybe try it a little more for even better performance. If the performance is not good, something different should be tried. In Learning Momentum, there are specific defined rules that govern parametric adjustments as well as situational identification characteristics that direct the adjustment policies in learning.

In previous use in reactive robotic systems, a number of appropriate and practical situations were pre-defined, which would be different depending on the nature of the robot's task. Taking a navigation task for example, a robot could find itself steadily moving toward its goal, having little, or no progress due to too many obstacles in the vicinity. Using a history of its sensor readings, the robot would determine which situation it is currently in, and appropriate adjustments would be applied to relevant behavioral parameters for that particular situation. Continuous adaptation of behavioral parameters in this manner was found to effectively reach a satisfactory solution.

For the Mood component, Learning Momentum is applied with some variations since the human-robot interaction aspect redefines the problem. The judgment of a robot's affective performance is inevitably subjective as viewed by the human interacting with it. In order for the robot to make appropriate adjustments to its affective performance to better align with the user's desires, the system needs a means to determine the human's satisfaction level or specific preferences. This could be achieved through vision or speech recognition technology for a more natural and complete robotic system, but for proof of concept in this study, a computer graphical user interface (GUI) suffices.

A set of questions are carefully crafted such that a naive user can effectively and intuitively convey his/her preference in terms of the robot's mood. For example, a question might ask if the user would like the robot's mood to be more positive or negative every morning. On the GUI, the questions are presented in a certain order requesting the user's input. When the user is finished with the sequence of questions, the responses are translated into a selection of low-level parametric adjustment rules that must be applied in order to reflect the user's opinions on the robot's affective performance.

An arbitrary number of adjustment rules can be flexibly formulated and linked to GUI questions with a configuration file. Through the file, an adjustment rule is explicitly declared with information such as to which specific moods and in which specific time step intervals (for cyclic moods) or resultant condition strength intervals (for situational and environmental moods) the adjustments apply, the adjustment values (in ranges) for the various parameters described in section III on the Mood component, etc. To generate the actual adjustment value applied to each parameter, a random value is selected from the defined adjustment range for the parameter. To decide on the appropriate adjustment values to use in making a certain mood more positive or negative, the psychological data on correlation between mood (positive and/or negative affect) and the five personality dimensions were taken into consideration [2] and serve as the basis for these alterations.

\section{IMPLEMENTATION AND SIMULATIONS}

The TAME framework has been implemented as a separate, stand-alone process (referred to as TAME Module) with Learning Momentum (referred to as LM Module). The influence of emotions on mood is not as yet incorporated in the Mood component. TAME Module has been integrated within MissionLab ${ }^{1}$, which is a robotic software toolset with a graphical user interface that allows an operator to easily create and configure multi-robot missions [25], [26]. As a test bed, Aldebaran Robotics' Nao humanoid platform is used. Since details on the software architecture of TAME Module and on the complete implementation is beyond the scope of this paper, only a brief explanation is provided in this section.

\section{A. MissionLab Overview}

Using Configuration Editor (cfgEdit), an operator can specify a robotic mission using a graphical representation called FSA, or finite state acceptor [27]. In FSA representation, various behaviors or actions to perform are combined together to create a mission, and the transition from one behavior to the next is triggered by certain perceptual conditions. The resulting mission, after being translated into $\mathrm{C}++$, is compiled to make Robot Executable. The compiled program, then, can be tested in MissionLab's simulation environment, or it can be deployed on real robotic platforms. When the robots are executing the mission, the operator can monitor its execution in real-time using mlab GUI display. HServer [26] is a hardware abstraction layer separate from Robot Executable, and it acts as a control interface to various robotics hardware. In MissionLab, HServer enables more flexible coordination with different robotic platforms.

\section{B. Integration with MissionLab}

For the integration, an interface has been added to HServer for controlling Nao at the hardware level. Through HServer, Robot Executable, which contains the actual control code for the robot's mission, can communicate the necessary control commands to Nao for execution.

HServer also continuously receives perceptual data from the robot and relays the information to both Robot Executable and TAME Module. Robot Executable needs the perceptual data for performing certain behaviors and determining when to make the transition between behaviors. HServer also organizes the perceptual data into stimuli information for the Emotion component (or condition information for the Mood component) in TAME Module.

\footnotetext{
${ }^{1}$ MissionLab is freely available for research and development and can be found at http://www.cc.gatech.edu/ai/robot-lab/research/MissionLab/
} 


\section{High-level Architectural View}

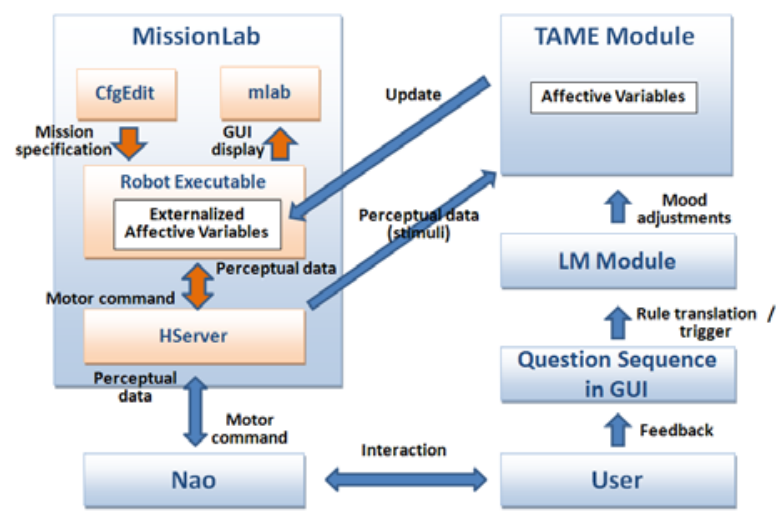

Fig. 3. High-level architectural view of the integration of TAME Module, LM Module and Nao with MissionLab.

Various configurations for TAME Module, such as the actual value for each personality dimension and various parameter values used for emotion generation, are defined using a configuration file. In assigning values for personality dimensions, a study on the Five Factor Model self-reported personality scores [28] is used as a guide.

In Robot Executable, there is a mirror database holding TAME Module's value for each personality and emotion dimension (the Mood and Attitude components do not have direct influence on the behavioral parameters of the robot). Continually being updated by TAME Module at three hertz (to ease computational burden), the personality and emotion information in Robot Executable, in turn, influences robot's behaviors by changing appropriate behavioral parameters.

For example, suppose there is a general walk behavior designed for $\mathrm{Nao}$ with parameters such as general gait speed and the amplitude of arm swinging. According to the information in the mirrored database, the parameters can be changed such that the same walk behavior portrays various walking styles with different affective characteristics.

Through the sequence of questions designed and presented in GUI, the user can communicate with LM Module, which in turn, adapts the Mood component in TAME Module to the user's preference. A graphical representation of the overall integration is shown in Fig. 3.

\section{Simulations}

A series of simulations were run to illustrate how Learning Momentum can be used to adapt the Mood component to the user's preference. The simulation settings were simplified so that the resulting influence was clear and straightforward to observe in a reasonable period of time. In this case, only a cyclic daily mood was defined, and the mood influence was absent (mood intensity values of all $0 \mathrm{~s})$ at the particular time step (in the morning) when the Learning Momentum interaction began. The dependency information as mentioned in (3) $\left(d p_{i, j}\right)$ were all set to have direct influence, the scaling factor as mentioned in (4) $(\mu)$ was set to 1 and the personality values were set to the corresponding mean personality scores
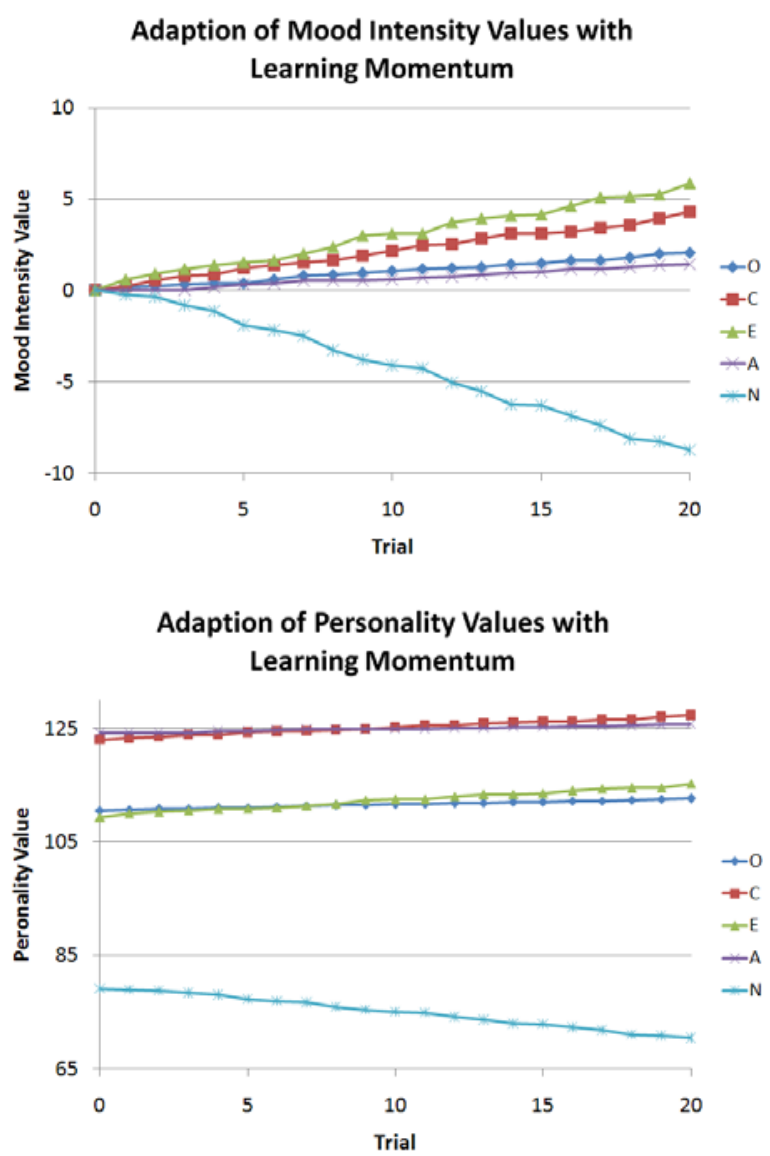

Fig. 4. On top is continuous Learning Momentum simulation trials and their influence on the mood intensity values for each personality dimension: Openness (O), Conscientiousness (C), Extroversion (E), Agreeableness (A), and Neuroticism $(\mathrm{N})$. The resulting bias on personality is shown on bottom.
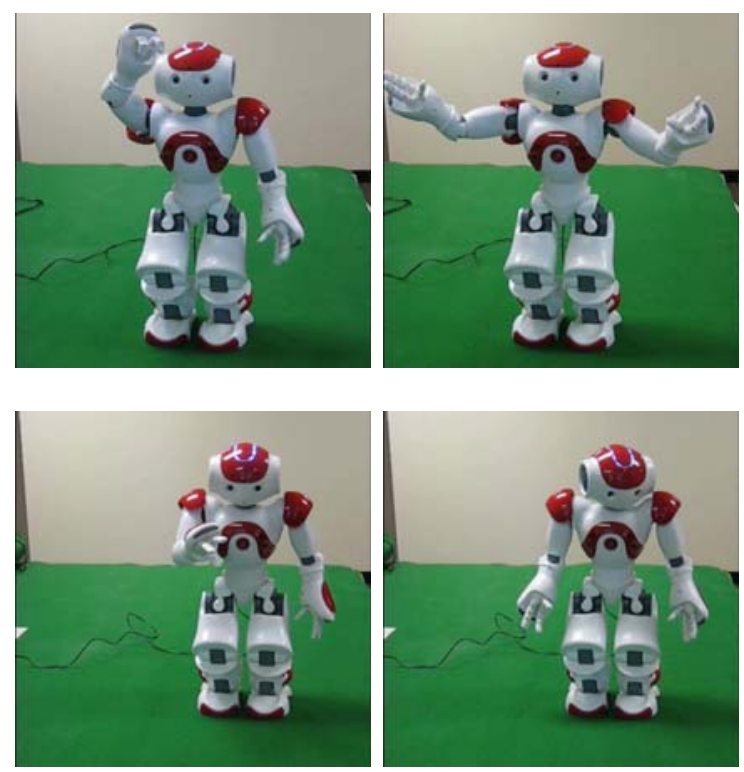

Fig. 5. Different affective characteristics for greeting behavior. The top images show Nao performing the behavior with high Extroversion level, and the bottom images show the behavior with low Extroversion level. 
as found in [28]. A specific rule was declared in the configuration file that would make the cyclic daily mood to be more positive in the morning. By repeatedly interacting with the question sequence GUI, that specific rule was repeatedly made to fire. The weighted filter average was configured so that the adjustments made by the rule would occur instantly. Using the correlation findings in [2] as a guide, the adjustment ranges (in which random adjustment values are selected) used to make the mood more positive were $0 \sim+0.2$ for $m_{\text {Openness, }}, 0 \sim+0.4$ for $m_{\text {Conscientiousness }}, 0 \sim$ +0.8 for $m_{\text {Extroversion }}, 0 \sim+0.2$ for $m_{\text {Agreeableness }}$, and $-0.8 \sim 0$ for $m_{\text {Neuroticism. }}$. The simulation results are shown in Fig. 4, and an example of resulting changes in a robotic behavior is shown in Fig. 5.

\section{CONCLUSION AND FUtURE WORK}

Based on an integrative affective model for robotic behavior, TAME, this paper presents its Mood component. A method of continuously adapting the Mood component to individual user's preference is described using a form of parametric adjustment termed Learning Momentum, and simulation results are provided for proof of concept. A formal human-robot interaction study will be conducted in the future to investigate humans' perception of various affective behaviors influenced by the Mood component and also to ascertain how effectively the component can be adapted to tailor the overall affective system (TAME) to individual user's preferences.

\section{REFERENCES}

[1] R. R. McCrae and P. T. Costa, "Toward a new generation of personality theories: theoretical contexts for the Five-Factor Model," in The Five-Factor Model of Personality, J. S. Wiggins, Ed. New York: Guilford, 1996, pp. 51-87.

[2] D. Watson, L. A. Clark, and A. Tellegen, Mood and Temperament. The Guilford Press, 2000, ch. 1, 3-4.

[3] L. Canamero, "Designing emotions for activity selection in autonomous agents," in Emotions in Humans and Artifacts, R. Trappl, P. Petta, and S. Payr, Ed. Cambridge, MA: MIT Press, 2003, pp. 115-148.

[4] S. J. Breckler and E. C. Wiggins, "On defining attitude and attitude theory: once more with feeling," in Attitude Structure and Function, A. R. Pratkanis, S. J. Breckler, and A. G. Greenwalk, Ed. Hillsdale, NJ: Erlbaum, 1989, pp. 407-429.

[5] C. Nass, J. Steuer, and E. R. Tauber, "Computers are social actors," in Proc. SIGCHI Conf. on Human Factors in Computing Systems: Celebrating Interdependence, Boston, 1994, pp. 72-78.

[6] L. Moshkina, "An integrative framework for affective agent behavior," in IASTED Int.Conf. on Intelligent Systems and Control, 2006.

[7] L. Moshkina, R. C. Arkin, J. Lee, and H. Jung, "Time-varying affective response for humanoid robots," in Proc. Int. Conf. on Social Robotics (ICSR), Seoul, 2009.

[8] L. Moshkina, "An integrative framework of time-varying affective robotic behavior," Ph.D. proposal, College of Computing, Georgia Institute of Technology, Atlanta, 2006.

[9] R. J. Clark, R. C. Arkin, and A. Ram, "Learning Momentum: on-line performance enhancement for reactive systems," in Proc. IEEE Int. Conf. on Robotics and Automation (ICRA), Nice, 1992, pp. 111-116.
[10] J. B. Lee and R. C. Arkin, "Learning Momentum: integration and experimentation," in Proc. IEEE Int. Conf. on Robotics and Automation (ICRA), Seoul, 2001, pp. 1975-1980.

[11] J. B. Lee, M. Likhachev, and R. C. Arkin, "Selection of behavioral parameters: integration of discontinuous switching via case-based reasoning with continuous adaptation via Learning Momentum," in Proc. IEEE Int. Conf. on Robotics and Automation (ICRA), Washington, D.C., 2002, pp. 1275-1281.

[12] J. B. Lee and R. C. Arkin, "Adaptive multi-robot behavior via Learning Momentum," in Proc. IEEE/RSJ Int. Conf. on Intelligent Robots and Systems (IROS), Las Vegas, 2003, pp. 2029-2036.

[13] T. Fong, I. Nourbakhsh, and K. Dautenhahn, "A survey of socially interactive robots," Robotics and Autonomous Systems, vol. 42, no. 3-4, pp. 143-166, Mar. 2003.

[14] C. Breazeal, "Emotion and sociable humanoid robots," International Journal of Human-Computer Studies, vol. 59, issues 1-2, pp. 119-155, Jul. 2003.

[15] K. Itoh, H. Miwa, Y. Nukariya, M. Zecca, H. Takanobu, S. Roccella, et al. "Behavior generation of humanoid robots depending on mood," in Proc. 9th Int. Conf. on Intelligent Autonomous Systems (IAS-9), Amsterdam, 2006, pp. 965-972.

[16] N. Kubota, Y. Nojima, N. Baba, F. Kojima, and T. Fukuda, "Evolving pet robot with emotional model," in Proc. of IEEE Congress on Evolutionary Computation, vol. 2, San Diego, 2000, pp.1231-1237.

[17] R. Gockley, R. Simmons, and J. Forlizzi, "Modeling affect in socially interactive robots," in Proc. IEEE Int. Symposium on Robot and Human Interactive Communication (RO-MAN), Hatfield, 2006, pp. 558-563.

[18] R. J. Davidson, "On emotion, mood and related affective constructs," in The Nature of Emotion: Fundamental Questions, 8th ed. vol. 4, D. Ekman, P. Ekman, and R. Davidson, Ed. New York: Oxford University Press, 1994.

[19] G. L. Clore, R. S. Wyer, Jr., B. Dienes, K. Gasper, C. Gohm, and L. Isbell, "Affective feelings as feedback: some cognitive consequences," in Theories of Mood and Cognition: A Users' Handboook, 1st ed., L. L. Martin and G. L. Clore, Ed. Psychology Press, 2001, pp. $27-62$.

[20] N. H. Frijda, "Varieties of affect: emotions and episodes, moods, and sentiments," in The Nature of Emotion: Fundamental Questions, 8th ed. vol. 4, D. Ekman, P. Ekman, and R. Davidson, Ed. New York: Oxford University Press, 1994.

[21] D. Watson and L. A. Clark, "Emotions, moods, traits, and temperaments: conceptual distinctions and empirical findings," in The Nature of Emotion: Fundamental Questions, 8th ed. vol. 4, D. Ekman, P. Ekman, and R. Davidson, Ed. New York: Oxford University Press, 1994.

[22] A. M. Isen, "Positive affect," in Handbook of Cognition and Emotion, 1st ed., T. Dalgleish and M. Power, Ed. Wiley, 1999, pp. 521-539.

[23] L. R. Goldberg, "An alternative 'description of personality': the Big Five Factor structure," Journal of Personality and Social Psychology, vol. 59, pp. 1216-1229, Dec. 1990.

[24] P. Ekman, "Moods, emotions and traits," in The Nature of Emotion: Fundamental Questions, 8th ed. vol. 4, D. Ekman, P. Ekman, and R. Davidson, Ed. New York: Oxford University Press, 1994.

[25] D. MacKenzie, R. Arkin, and J. Cameron, "Multiagent mission specification and execution," Autonomous Robots, vol. 4, no. 1, pp. 29-52, Mar. 1997.

Georgia Tech Mobile Robot Laboratory, Manual for MissionLab Version 7.0, 2007. Available: h ttp://www.cc.gatech.edu/ai/robot-lab /research/MissionLab/

[26] R. Arkin, Behavior-Based Robotics. Cambridge, MA: MIT Press, 1998.

[27] P. T. Costa and R. R. McCrae, NEO PI-R Professional Manual. Odessa, FL: Psychological Assessment Resources, 1992. 